\title{
Outcomes of Primary Unilateral Cheiloplasty in Same-Day Surgical Settings
}

\author{
Mansoor Khan ${ }^{1}$, Hidayat Ullah ${ }^{2}$, Asif Aziz ${ }^{1}$, Muhammad Tahir ${ }^{2}$ \\ ${ }^{1}$ Plastic and Burn Surgery Unit, Khyber Teaching Hospital, Peshawar; ${ }^{2}$ Plastic and Reconstructive Surgery Department, Hayatabad Medical \\ Complex, Peshawar, Pakistan
}

Background Financial, clinical, and psychological considerations have made same-day surgery an attractive option for a variety of procedures. This article aimed to analyse the postoperative results of same-day primary unilateral cleft nasolabial repair.

Methods This study was performed from 2011 to 2014. Unilateral cleft lip patients fulfilling the inclusion criteria were preoperatively classified as mild, moderate, and severe. All patients underwent same-day surgery and were discharged after satisfying the appropriate clinical criteria, receiving thorough counselling, and the establishment of a means of communication by phone. Postoperative outcomes were assessed and stratified according to preoperative severity and the type of repair.

Results A total of 423 primary unilateral cleft lip patients were included. Fisher's anatomical subunit approximation technique was the most common procedure, followed by Noordhoff's technique. The postoperative outcome was good in $89.8 \%$ of cases, fair in $9.9 \%$ of cases, and poor in $0.2 \%$ of cases. The complication rate was $1.18 \%(n=5)$, and no instances of mortality were observed. The average hospital stay was 7.5 hours, leading to a cost reduction of $19 \%$ in comparison with patients who stayed overnight for observation.

Conclusions Mild unilateral cleft lip was the most common deformity for which Fisher's anatomical subunit approximation technique was performed in most of the cases, with satisfactory postoperative outcomes. Refinements in the cleft rhinoplasty techniques over the course of the study improved the results regarding cleft nasal symmetry. Single-day primary unilateral cleft cheiloplasty was found to be a cost-effective procedure that did not pose an additional risk of complications.

Keywords Cleft lip / Rhinoplasty / Day care
Correspondence: Mansoor Khan Plastic and Burns Surgery, Khyber Teaching Hospital, Peshawar, Pakistan PO Box 25000

Tel: $+92-346-9641432$

Fax: +92-919-216340

E-mail:drkhanps@yahoo.com

We would like to thank Dr. Alamzeb Khan for his extraordinary support and for contributing the illustrations.

No potential conflict of interest relevant to this article was reported.

Received: 14 Aug 2015 • Revised: 26 Jan 2016 • Accepted: 15 Feb 2016

pISSN: 2234-6163 • elSSN: 2234-6171 • http://dx.doi.org/10.5999/aps.2016.43.3.248 • Arch Plast Surg 2016;43:248-253

\section{INTRODUCTION}

Unilateral cleft lip is one of the most common congenital craniofacial deformities. Its incidence is highest in Asians (1/500), followed by Caucasians $(1 / 1,000)$ and Africans $(1 / 2,500)[1,2]$. The reported incidence in Pakistan is 1.46 per 1,000 live births [3].
Since it is a complex deformity, numerous cleft cheiloplasty techniques have been devised, with no single technique universally recognized as optimal. Assessing postoperative outcomes is the most important aspect of improving surgical techniques by determining their advantages and disadvantages [4]. Evaluating the aesthetic outcomes of unilateral nasolabial repair is very challenging, due to the mandatory consideration of preopera- 
tive severity and the existence of multiple postoperative nasolabial anthropometric parameters [5].

Single-day surgery is becoming more common for an increasing number of procedures in different surgical specialities because it has financial benefits, leads to fewer surgical site infections, expedites patients' postoperative recovery in the home environment, and avoids the psychological effects of hospitalization [6]. Plastic surgery procedures, especially cleft lip and palate repair, have been no exception to this trend for single-day surgery to become more common [7].

The aim of the present study was to evaluate the preoperative severity and postoperative outcomes of single-day primary unilateral cleft nasolabial repair, with the objective of shortening the patients' hospital stay and decreasing their economic burden on the healthcare facility.

\section{METHODS}

After approval from the ethical committee, this descriptive crosssectional study was conducted from January 2011 to December 2014. All patients who presented for primary unilateral cleft lip repair were initially included irrespective of their gender. Patients were then excluded if they were less than three months of age, displayed syndromic associations and co-morbidities, did not have competent parents or relatives, were psychologically unstable, or lived far from the hospital. After providing informed consent regarding the study protocol, the patients were thoroughly assessed through a history and clinical examination by the two primary surgeons and the two anaesthesiologists. Preoperatively, patients were restricted from consuming food or drink orally according to guidelines of the American Society of Anesthesiologists [8]. Clinical photographs were acquired preoperatively and up to six months postoperatively in order to evaluate the results. Preoperatively, patients were categorized on the basis of the severity of the cleft [9] as follows: (1) Mild: Incomplete cleft lip. (2) Moderate: Complete but not wide cleft lip, with some tissue contact between the lateral and medial lip segments at rest. (3) Severe: Complete and wide cleft lip, without tissue contact between the medial and lateral lip segments at rest.

Lip repair with primary rhinoplasty was performed under general anaesthesia (Fig. 1). Postoperatively, patients were observed in the recovery room and their vitals were monitored. Patients were then discharged home after achieving full alertness and ambulation, reporting that they were not in pain, and starting alimentation. Patients were discharged under the supervision of at least one mentally and physically capable escort who could drive him/her home and supervise him/her for 24 hours with clear verbal and/or written instructions for contacting the surgical staff in case of pain, vomiting (refractory to the prescribed medications), bleeding, fever, infection, or other concerns.

The postoperative results were subjectively graded as good, fair, and poor (Table 1) [9]. The data were evaluated and stratified according to the severity of the cleft lip and the repair technique.

Table 1. Outcome evaluation criteria [9]

\begin{tabular}{|lccc|}
\hline Features & Unsatisfactory Satisfactory & Good \\
\hline Symmetry of Cupid's bow & 0 & 1 & 2 \\
Symmetry of the lateral lip & 0 & 1 & 2 \\
Symmetry of the nose & 0 & 1 & 2 \\
Symmetry of the free vermillion & 0 & 1 & 2 \\
Symmetry of the wet/dry vermillion & 0 & 1 & 2 \\
\hline Outcome score: good, 8-10; fair, 5-7; poor, 0-4. & & \\
\end{tabular}

\section{Fig. 1. Surgical techniques of unilateral cleft lip repair}

(A) Fisher's anatomical subunit approximation technique. (B) Noordhoff's technique. (C) Primary rhinoplasty with an inverted-U semi-open rhinoplasty incision and Tajima suspension stitch.

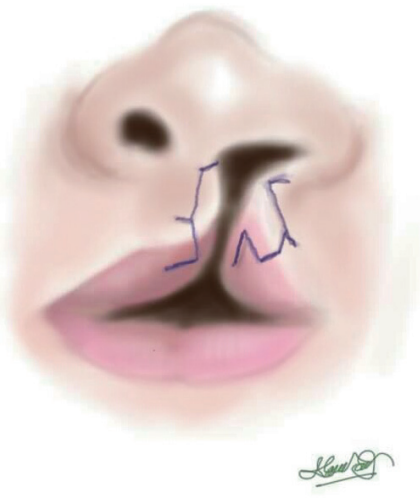

(A)

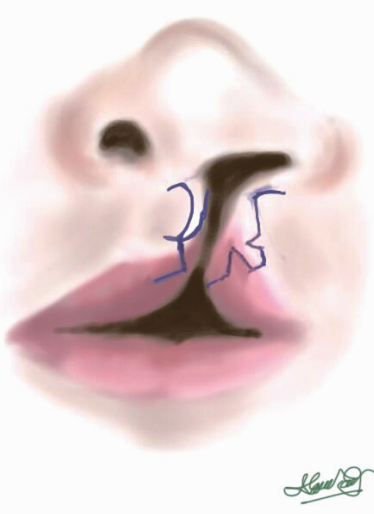

(B) 


\section{Fig. 2. Mild left unilateral cleft lip repair}

(A) Mild left unilateral cleft lip. (B) Four-week postoperative follow-up with good results. (C) Follow-up after six months.
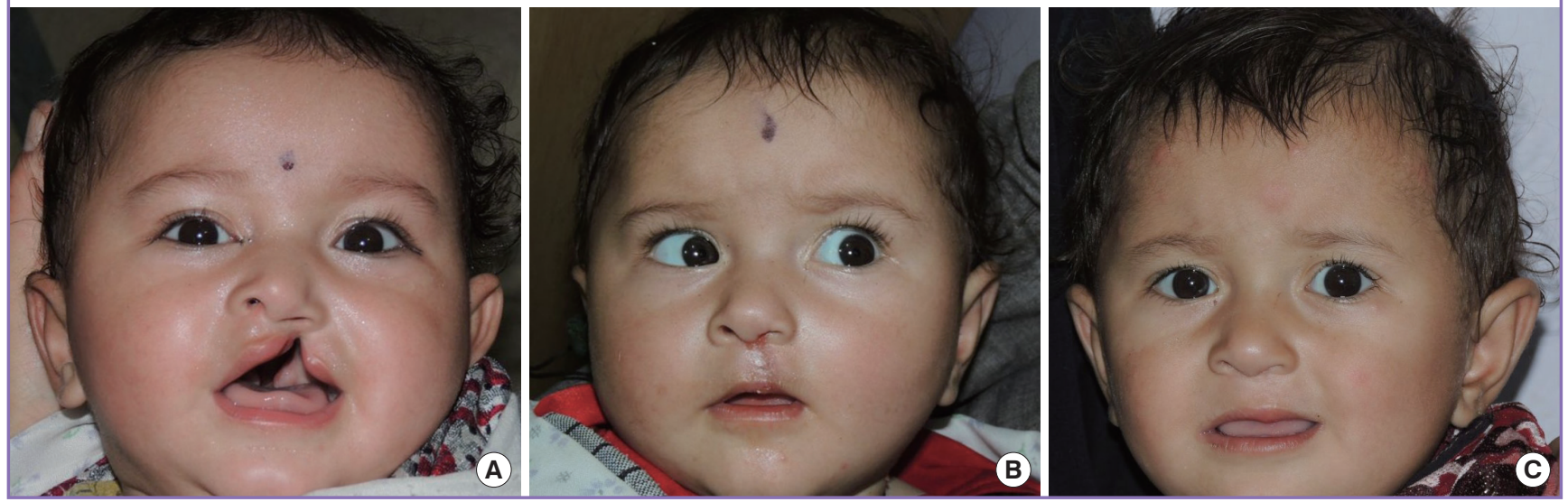

Fig. 3. Severe left unilateral cleft lip repair

(A) Severe left unilateral cleft lip. (B) Left unilateral cheiloplasty using Noordhoff's technique with good results (four-week postoperative followup). (C) Fifteen-month postoperative follow-up.
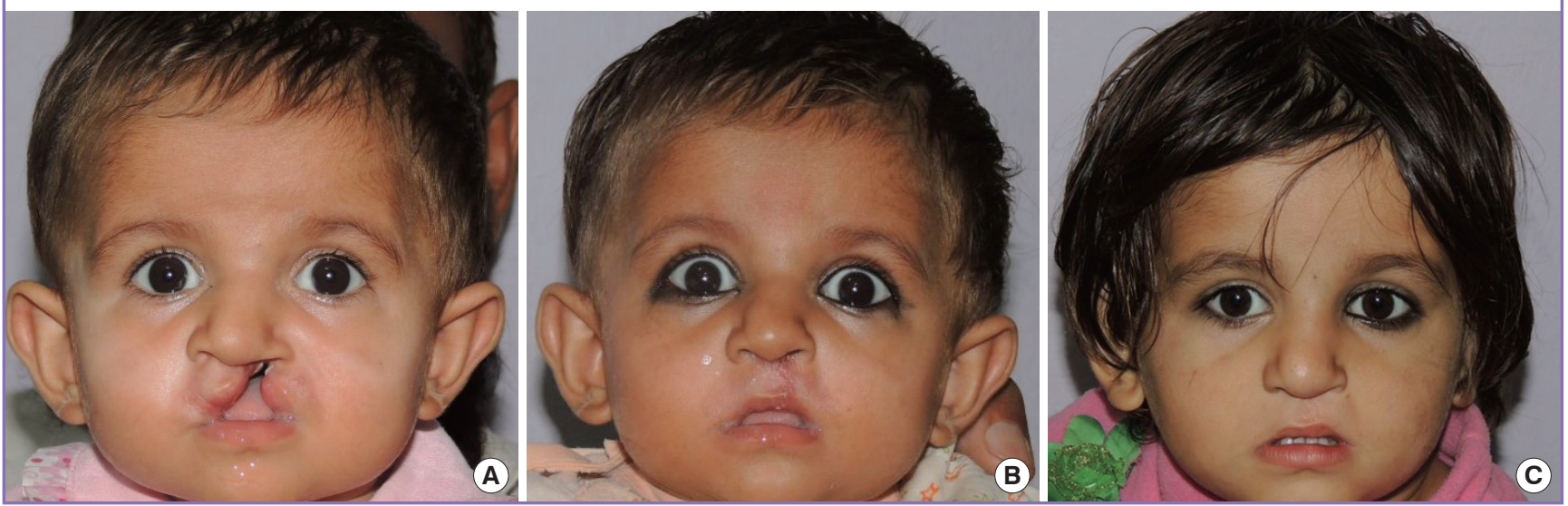

Table 2. Cleft lip severity stratified versus the surgical procedure performed

\begin{tabular}{|lcccccc|}
\hline \multirow{2}{*}{$\begin{array}{l}\text { Severity of } \\
\text { cleft lip }\end{array}$} & \multicolumn{5}{c}{ Surgical procedure } \\
\cline { 2 - 6 } & Fisher's repair & Mohler's repair & Noordhoff's repair & Rose Thompson's repair & Millard repair & Total \\
\hline Mild & 180 & 0 & 8 & 2 & 0 & $190(44.9)$ \\
Moderate & 55 & 0 & 7 & 0 & $63(14.9)$ \\
Severe & 141 & 1 & 25 & 0 & 1 & 3 \\
Total, $n(\%)$ & $376(88.9)$ & $1(0.24)$ & $40(9.45)$ & $2(0.47)$ & $4(0.94)$ \\
\hline
\end{tabular}

\section{RESULTS}

A total of 423 primary unilateral cleft lip repairs were performed, including 265 (62.6\%) in males and 158 (37.4\%) in females. The patients ranged in age from three to 384 months (mean, $21.4 \pm$ 4.2 months). Mild, moderate, and severe primary unilateral cleft lip occurred in 190 (44.9\%), 63 (14.9\%), and 170 (40.2\%) patients, respectively. Fisher's anatomic subunit approximation technique was the most commonly performed procedure (per- formed in $88.9 \%$ of cases), followed by Noordhoff's technique (Table 2). We determined that 380 patients (89.8\%) had good outcomes, 42 (9.9\%) had fair outcomes, and one $(0.2 \%)$ had a poor outcome (Figs. 2-5). The symmetry of the nose was good in $57.4 \%$ of cases $(n=243)$, satisfactory in $38.3 \%$ of patients $(n=162)$, and unsatisfactory in $4.3 \%$ of cases $(n=18)$ (Table 3$)$. Over the study period, a trend of overall improvement in postoperative outcomes was observed, but we did not find it to be statistically significant (Table 4, Figs. 6, 7). The rate of complica- 


\section{Fig. 4. Mild left unilateral cleft lip repair}

(A) Preoperative image of left unilateral cleft lip. (B) Four-week postoperative image with good results. (C) Six-month follow-up.
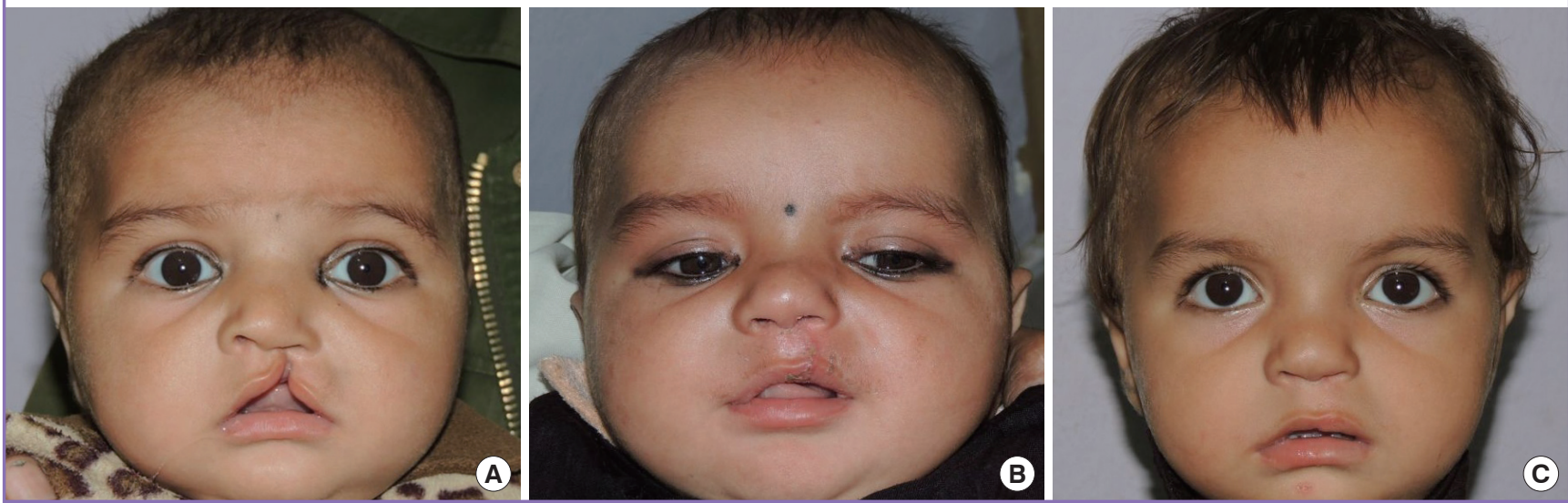

\section{Fig. 5. Severe left unilateral cleft lip repair}

(A) Severe left unilateral cleft lip. (B) Four-weeks postoperative follow-up. (C) Six-month postoperative follow-up with good results.
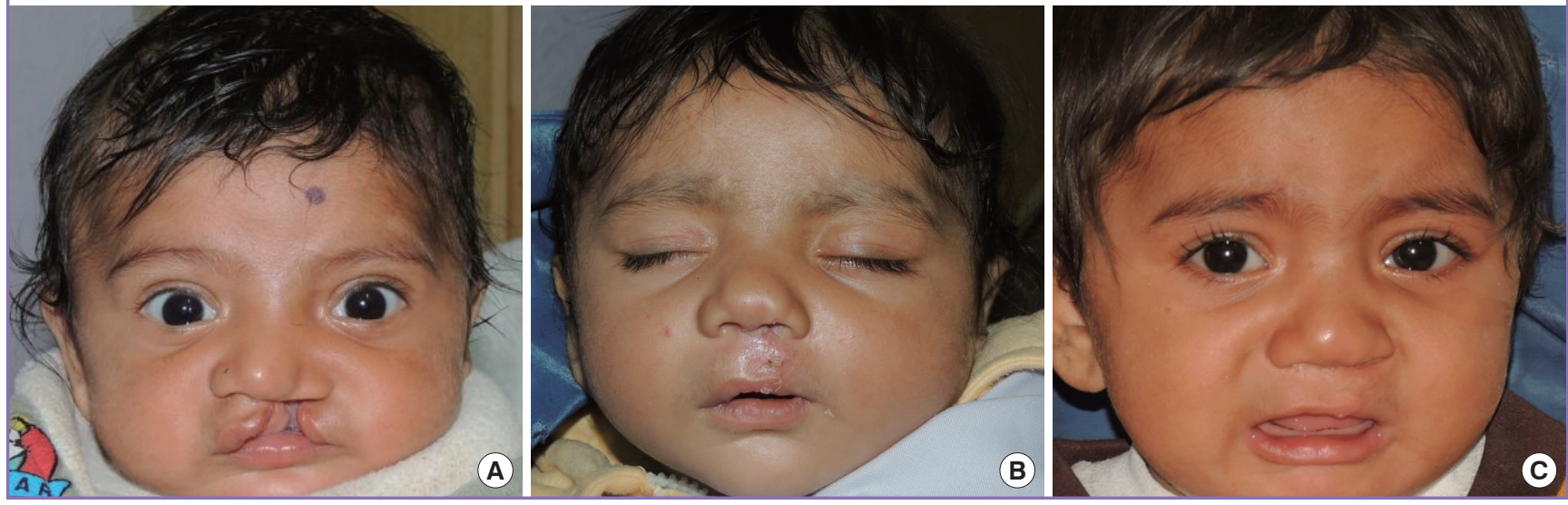

Table 3. Distribution of postoperative nasal symmetry outcomes over time

\begin{tabular}{|lcccc|}
\hline \multirow{3}{*}{ Year } & \multicolumn{3}{c}{ Nasal symmetry } & \multirow{2}{*}{ Total } \\
\cline { 2 - 4 } & Unsatisfactory & Satisfactory & Good & \\
\hline 2011 & $6(9.8)$ & $27(44.3)$ & $28(45.9)$ & 61 \\
2012 & $8(6.4)$ & $60(48.0)$ & $57(45.6)$ & 125 \\
2013 & $2(1.6)$ & $51(40.1)$ & $74(58.3)$ & 127 \\
2014 & $2(1.8)$ & $24(21.8)$ & $84(76.4)$ & 110 \\
Total & 18 & 162 & 243 & 423 \\
\hline Values are presented as or number (\%). & \\
\hline
\end{tabular}

tions was $1.18 \%(\mathrm{n}=5)$ and no cases of mortality were observed. The most common complication was stitch sinus $(0.71 \%)$, followed by nasal hematoma $(0.24 \%)$, and severe lower respiratory tract infection $(0.24 \%)$.

\section{Cleft severity and surgical outcomes}

One patient with severe unilateral cleft lip had a poor outcome,
Table 4. Distribution of postoperative outcomes of primary unilateral cleft lip repair over time

\begin{tabular}{|c|c|c|c|c|c|}
\hline \multirow{2}{*}{ Outcome } & \multicolumn{4}{|c|}{ Year } & \multirow{2}{*}{ Total } \\
\hline & 2011 & 2012 & 2013 & 2014 & \\
\hline Poor & $1(1.64)$ & 0 & 0 & 0 & 1 \\
\hline Fair & $5(8.2)$ & $15(12)$ & $17(13.4)$ & $5(4.54)$ & 42 \\
\hline Good & $55(90.2)$ & 110 (88) & $110(86.61)$ & 105 (95.5) & 380 \\
\hline Total & 61 & 125 & 127 & 110 & 423 \\
\hline
\end{tabular}

while $88.1 \%$ of cases $(n=37)$ with a fair outcome belonged to the severe group (Table 5). The symmetry of Cupid's bow was good in $86.1 \%$ of cases $(n=364)$, satisfactory in $13 \%$ of cases $(\mathrm{n}=55)$, and unsatisfactory in $0.9 \%$ of cases $(\mathrm{n}=4)$ (Table 6$)$.

\section{Type of cleft lip repair and outcomes}

Of the patients in the Fisher's anatomic subunit approximation 


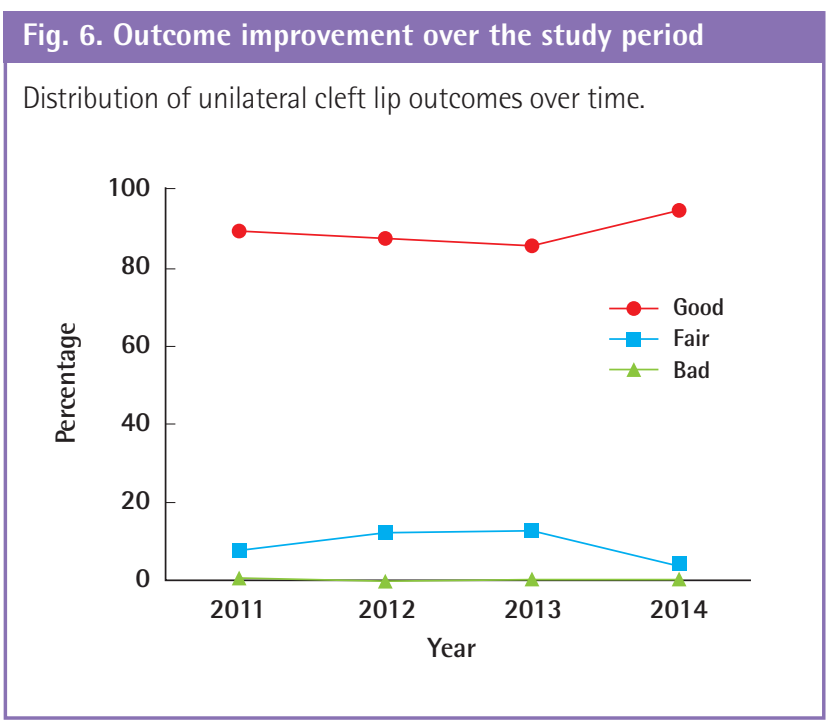

Table 5. Cleft lip severity versus postoperative outcomes

\begin{tabular}{|lrrrr|}
\hline \multirow{2}{*}{$\begin{array}{l}\text { Postoperative } \\
\text { outcome }\end{array}$} & \multicolumn{3}{c}{ Severity of cleft lip } & \multirow{2}{*}{ Total } \\
\cline { 2 - 4 } & Mild & Moderate & Severe & \\
\hline Poor & 0 & 0 & 1 & 1 \\
Fair & 3 & 2 & 37 & 42 \\
Good & 187 & 61 & 132 & 380 \\
Total & 190 & 63 & 170 & 423 \\
\hline
\end{tabular}

\section{Fig. 7. Nasal symmetry correction outcome}

Improvements in the symmetry achieved by nasal deformity corrections over the study period.

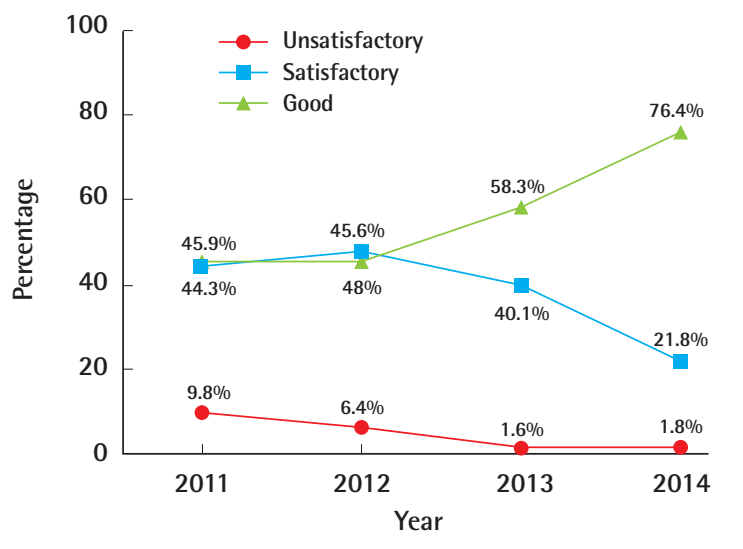

Table 6. Cleft lip severity versus postoperative Cupid's bow symmetry

\begin{tabular}{|lrccr|}
\hline Postoperative & \multicolumn{3}{c}{ Severity of cleft lip } & Total \\
\cline { 2 - 4 } $\begin{array}{c}\text { Cupid's bow } \\
\text { symmetry }\end{array}$ & Mild & Moderate & Severe & \\
\hline Unsatisfactory & 0 & 0 & 4 & 4 \\
Satisfactory & 9 & 5 & 41 & 55 \\
Good & 181 & 58 & 125 & 364 \\
Total & 190 & 63 & 170 & 423 \\
\hline
\end{tabular}

Table 7. Surgical procedure versus postoperative Cupid's bow symmetry

\begin{tabular}{|c|c|c|c|c|c|c|}
\hline \multirow{2}{*}{$\begin{array}{l}\text { Symmetry of } \\
\text { Cupid's bow }\end{array}$} & \multicolumn{5}{|c|}{ Surgical procedure } & \multirow[b]{2}{*}{ Total } \\
\hline & $\begin{array}{l}\text { Fisher's } \\
\text { repair }\end{array}$ & $\begin{array}{l}\text { Mohler's } \\
\text { repair }\end{array}$ & $\begin{array}{l}\text { Noordhoff's } \\
\text { repair }\end{array}$ & $\begin{array}{l}\text { Rose Thompson's } \\
\text { repair }\end{array}$ & $\begin{array}{l}\text { Millard } \\
\text { repair }\end{array}$ & \\
\hline Unsatisfactory & 4 & 0 & 0 & 0 & 0 & 4 \\
\hline Satisfactory & 51 & 1 & 0 & 1 & 2 & 55 \\
\hline Good & 321 & 0 & 40 & 1 & 2 & 364 \\
\hline Total & 376 & 1 & 40 & 2 & 4 & 423 \\
\hline
\end{tabular}

technique group, $89.4 \%(n=336)$ had good overall outcomes and $10.6 \%$ of patients $(n=40)$ had satisfactory outcomes, while good Cupid's bow symmetry was found in $85.4 \%(n=321)$, satisfactory symmetry in $13.6 \%(n=51)$, and unsatisfactory symmetry in $1.1 \%$ of cases $(n=4)$ (Table 7$)$. All of the patients $(100 \%)$ who were treated using Noordhoff's technique had good outcomes and good Cupid's bow symmetry.

\section{Nasal symmetry}

Over the study period, an overall improvement in the achievement of good symmetry in cleft nasal deformities was observed (45.9\%-76.4\%) and the rate of unsatisfactory nasal symmetry decreased from $9.5 \%$ to $1.8 \%$ (Table 7 , Fig. 7 ).

\section{Cost-effectiveness of single-day lip repair surgery}

The average hospital stay was 7.5 hours (range, 6-9 hours). The hospital cost was reduced by $19 \%$ (3,800 PKR) in comparison to the costs incurred by patients who were observed overnight. The readmission rate was $0.24 \%(n=1)$ for severe lower respiratory tract infection.

\section{DISCUSSION}

Over time, measurements of surgical outcomes led to the current sophisticated repair techniques for cleft lip deformities. In this study we assessed the outcomes of primary unilateral cleft lip repair performed at our centre. The mean age of presentation for primary unilateral cleft lip repair was $21.4 \pm 4.2$ months, with 
a male-to-female ratio of 1.7:1. Abdurrazaq et al. [4] reported a similar delay in presentation and male-to-female ratio for unilateral cleft lip repair. The delayed presentation for lip repair may have been due to a lack of awareness, a lack of access to healthcare, and financial constraints.

At the time of presentation, the most common unilateral cleft lip types were mild (44.9\%) and severe (40.2\%), in contrast to Rajanikanth et al. [10], who reported that $75 \%$ of their cases were severe and only $25 \%$ were mild. Mortier et al. [11] reported that $44.2 \%$ of the cases were severe and $48.8 \%$ were moderate in their series. These difference may be explained by differences in the geographical distribution of these cases, the availability of nasoalveolar moulding, and the lack of a standardised scoring system.

The most commonly performed procedure for primary unilateral cleft lip repair in our patients was Fisher's anatomic subunit approximation technique, followed by Noordhoff's technique. Most of our patients had good postoperative results. Mortier et al. [11] reported good results in approximately $79.1 \%$ of cases and poor results in $4.6 \%$ of cases in whom Millard's rotation advancement technique was used. Abdurrazaq et al. [4] reported their postoperative assessment of their cases as good, fair, and poor in $67.9 \%, 28.4 \%$, and $3.7 \%$ of cases, respectively. They performed the Tennison-Randall and Millard techniques in $42.4 \%$ and $31.5 \%$ of the cases, respectively. In another series, Ajmal et al. [12] reported that Fisher's anatomic subunit technique led to good results in most of the population of their study. Among our patients, most of the fair and unsatisfactory results were found in patients with relatively severe cleft lip deformities. We observed improvement in the postoperative score for nasal symmetry over the study period due to refinements in the primary cleft rhinoplasty technique. In our experience, Noordhoff's technique showed better postoperative outcomes than Fisher's anatomic subunit approximation technique, but our results in this regard are inconclusive due to the limited number of patients in the former group. We observed a cost reduction of $19 \%$ for sameday surgery in our series, compared to the reductions of $15 \%$ $30 \%$ and $40 \%$ that have been reported for the USA and UK, respectively [6].

Randomized controlled trials are recommended to reach secure conclusions regarding these issues. In our experience, single-day unilateral cleft lip surgery was cost-effective and did not increase the rate of complications. The major limitation of our study is that we were unable to analyse the long-term results of our patients.

\section{REFERENCES}

1. Shapira Y, Haklai Z, Blum I, et al. Prevalence of non-syndromic orofacial clefts among Jews and Arabs, by type, site, gender and geography: a multi-center study in Israel. Isr Med Assoc J 2014;16:759-63.

2. Kling RR, Taub PJ, Ye X, et al. Oral clefting in china over the last decade: 205,679 patients. Plast Reconstr Surg Glob Open 2014;2:e236.

3. Elahi MM, Jackson IT, Elahi O, et al. Epidemiology of cleft lip and cleft palate in Pakistan. Plast Reconstr Surg 2004;113: 1548-55.

4. Abdurrazaq TO, Micheal AO, Lanre AW, et al. Surgical outcome and complications following cleft lip and palate repair in a teaching hospital in Nigeria. Afr J Paediatr Surg 2013; 10:345-57.

5. Molsted K. Treatment outcome in cleft lip and palate: issues and perspectives. Crit Rev Oral Biol Med 1999;10:225-39.

6. Wig J. The current status of day care surgery: a review. Indian J Anaesth 2005;49:459-.

7. Wood BC, Boyajian MJ, Zurakowski D, et al. Evaluating the need for routine admission following primary cleft palate repair: an analysis of 100 consecutive cases. Plast Reconstr Surg 2015; 136:502e-510e.

8. American Society of Anesthesiologists Committee. Practice guidelines for preoperative fasting and the use of pharmacologic agents to reduce the risk of pulmonary aspiration: application to healthy patients undergoing elective procedures: an updated report by the American Society of Anesthesiologists Committee on Standards and Practice Parameters. Anesthesiology 2011;114:495-511.

9. Bermudez L. Ciruestetic [Internet]. Bogota: Cirugia plastic; 2013 [cited 2015 Oct 16]. Available from: http://www.ciruestetic.com/resources/nasolabiaeval2013red.pdf.

10. Rajanikanth BR, Rao KS, Sharma SM, et al. Assessment of deformities of the lip and nose in cleft lip alveolus and palate patients by a rating scale. J Maxillofac Oral Surg 2012;11:3846.

11. Mortier PB, Martinot VL, Anastassov Y, et al. Evaluation of the results of cleft lip and palate surgical treatment: preliminary report. Cleft Palate Craniofac J 1997;34:247-55.

12. Ajmal S, Khan MA, Khan AT, et al. Evaluating anatomical subunit approximation technique for unilateral cleft lip repair. J Postgrad Med Inst 2010;24:68-72. 